\title{
Effects of weight loss and exercise on trunk muscle composition in older women
}

\author{
This article was published in the following Dove Press journal: \\ Clinical Interventions in Aging \\ 3 March 2014 \\ Number of times this article has been viewed
}

\begin{abstract}
Alice S Ryan
Aruna Selina Harduarsingh-

Permaul

Baltimore Veterans Affairs Medical Center; Gerontology and Geriatric Medicine, School of Medicine, University of Maryland; Baltimore Geriatric Research Education and Clinical Center (GRECC), and Research and Development Service, VA Maryland Health Care System, Baltimore, MD, USA
\end{abstract}

Background: Aging and obesity increase the risk for mobility limitations in women. Although trunk muscle composition is important to physical function, the implication of ectopic fat in the trunk muscles with respect to physical fitness and its potential for modification by lifestyle changes is unknown.

Methods: The effects of a 6-month period of either weight loss (WL) alone or of aerobic exercise (AEX) plus WL (AEX+WL), on trunk body composition, as measured by dual-energy $\mathrm{X}$-ray absorptiometry (DXA) (to measure trunk fat and lean mass) and computed tomography (CT) (to measure the erector spinae, psoas, lateral abdominal, rectus abdominis muscle, and intramuscular fat, and the intramuscular adipose tissue [IMAT] areas) was determined in 65 overweight and obese postmenopausal women (aged 50-76 years).

Results: The area of the erector spinae, psoas, and rectus abdominis muscles declined with age in the women $(P<0.05)$. Both the spinal and abdominal muscle areas were related to the maximal oxygen uptake $\left(\mathrm{VO}_{2} \max \right)(P<0.05)$. Body weight decreased by $8 \%$ after both $\mathrm{AEX}+\mathrm{WL}$ and WL $(P<0.001)$. The $\mathrm{VO}_{2}$ max increased by $11 \%$ after AEX+WL $(P<0.001)$ but did not change with WL alone (group effect, $P<0.001$ ). The DXA-measured trunk fat mass decreased by $16 \%$ after AEX+WL $(P<0.001)$ and by $12 \%$ after WL $(P<0.001)$. When both groups were combined, the IMAT decreased in all four muscle groups - by $6 \%$ in the erector spinae $(P<0.01)$, by $9 \%$ in the psoas $(P<0.01)$, by $11 \%$ in the lateral abdominals $(P<0.001)$, and by $6 \%$ in the rectus abdominis $(P<0.05)$. The loss of fat mass was related to the loss of IMAT of the erector spinae and the lateral abdominals.

Conclusions: A lifestyle modification of diet-induced WL alone, or with AEX training, results in a significant reduction of the fat infiltration in the abdominal and spinal muscles of the trunk region. Our finding that losses of total body fat predict a reduction in the IMAT of the trunk supports the idea that WL reduces fat depots throughout the body.

Keywords: dieting, intramuscular fat, aging, mobility, obesity

\section{Introduction}

Over two-thirds of postmenopausal women are overweight or obese. ${ }^{1}$ Older women are adversely affected by mobility limitations, which are predictive of disability. ${ }^{2}$ Moreover, overweight and obesity increases the risk of physical disability in middleaged and older women. ${ }^{3,4}$ In a 7-year follow-up study, women who were overweight or obese had an increased risk of mobility limitation, as determined by difficulty walking $1 / 4$ mile $(0.402 \mathrm{~km})$ or climbing stairs, compared with women of normal weight. ${ }^{5}$ Thus, the change in body composition with age and the accompanied reduction in physical fitness appear to be a detriment to function and mobility in older women.
Division of Gerontology and Geriatric Medicine, BT/I8/GR, $10 \mathrm{~N}$ Greene St, Baltimore Veterans Affairs Medical Center, Baltimore, MD 2120I, USA

$\mathrm{Tel}+\mathrm{I} 4106057 / 85$

Fax + I 4106057913

Email aryan@grecc.umaryland.edu 
Although fat infiltration in leg skeletal muscle has been associated with metabolic risk, ${ }^{6}$ adipose tissue deposition in the trunk muscles in older individuals may be important in physical function, in that low muscle density in the trunk muscles is related to poor physical function and back pain in both men and women. ${ }^{7}$ Furthermore, trunk muscle stability is integral to the performance of activities of daily living. Lower levels of trunk muscle attenuation and higher levels of intramuscular fat have been associated with reduced functional capacity in healthy older adults. ${ }^{7,8}$ Additionally, low spine muscle density, in both men and women, has been associated with hyperkyphosis, ${ }^{9}$ which reduces mobility and impairs balance. ${ }^{10,11}$ It is unknown whether trunk muscle composition is associated with physical fitness and whether it can be modified by lifestyle changes.

In light of these emerging indications that trunk muscle composition is important to physical function, as well as our limited knowledge of the effects of lifestyle modifications on trunk muscle composition, we conducted the present study, as a secondary analysis of a trial investigating skeletal muscle insulin sensitivity, ${ }^{12}$ and sought to 1 ) examine the relationships between trunk muscle composition and physical fitness, in sedentary, overweight, and obese African-American and Caucasian postmenopausal women, and 2) to determine the effects of 6 months of diet-induced weight loss (WL), alone and in combination with aerobic exercise (AEX) training, on trunk muscle composition. We hypothesized that trunk muscle area would be associated with physical fitness and that WL, alone or combined with AEX training, would reduce the fatty infiltration in trunk muscles in older women.

\section{Methods}

\section{Participants}

Postmenopausal women between 50-76 years of age who were healthy, and overweight or obese (body mass index [BMI] $>25 \mathrm{~kg} / \mathrm{m}^{2}$ [range $25-46 \mathrm{~kg} / \mathrm{m}^{2}$ ]) participated and were a part of a larger study. ${ }^{12}$ The participants were nonsmokers, weight stable $(<2.0 \mathrm{~kg}$ weight change in past year) and sedentary ( $<20$ minutes of aerobic exercise, twice per week). The women were screened with a medical history questionnaire, physical examination, and showed no evidence of cancer, liver, renal or hematological disease, or other medical disorders. All subjects underwent a screening graded treadmill test to exclude those with asymptomatic coronary artery disease. A total of 65 women met the study criteria $(62 \%$ Caucasian $[n=40]$ and $38 \%$ African-American [ $\mathrm{n}=25])$, completed the AEX+WL $(n=43)$ or WL $(n=22)$ interventions, and had trunk muscle composition measurements performed using dual-energy X-ray absorptiometry (DXA) (Prodigy, LUNAR Radiation Corp., Madison, WI, USA) and computed tomography (CT) (Siemens Somatom Sensation 64 Scanner; Siemens AG; Fairfield, CT, USA), which have not been previously described for publication. CT scans were not used for the analysis in those cases where the woman did not have a CT scan due to a scheduling conflict or where the images were no longer available for analysis. The Institutional Review Board of the University of Maryland approved all the study methods and procedures. Each participant provided written, informed consent to participate in the study.

\section{Study design}

The women received instruction in maintaining a weightstable, Therapeutic Lifestyle Changes (TLC) diet, ${ }^{13}$ from a registered dietitian 1 day/week for 6-8 weeks in order to maintain the dietary composition across the study: the subjects were already "weight stable" on the TLC diet at the baseline testing and were instructed to maintain the TLC diet throughout the study. After the baseline testing, the women attended weekly WL classes for 6 months for diet instruction led by the registered dietitian. Compliance with the diet was monitored using 7-day food records developed by the American Diabetes Association as part of the Exchange List System. To generate a caloric deficit, the dietitian estimated the subjects' total energy expenditure (TEE), using the Harris Benedict ${ }^{14}$ equation, to estimate the basal energy expenditure (BEE) and multiplied this number by an activity factor (AF) and thermic effect of food $(\mathrm{TEF})$, for eg, $\mathrm{TEE}=(\mathrm{BEE} \times$ sedentary AF of 1.25) $\times$ TEF of 1.1. To induce weight loss, 350-500 kcal was subtracted from the TEE. The research participant received instruction on a personalized food pattern for their WL calorie level. The pattern followed the format of the American Diabetes Exchange List System and provided 5 full days of menu ideas. The subjects in the AEX+WL group also exercised at the Baltimore Veterans Affairs (VA) Medical Center Geriatric Research Education and Clinical Center (GRECC) exercise facility three times per week for 6 months, using treadmills and elliptical trainers. Each exercise session included a 5-10 minute stretching and warm-up phase and a 5-10 minute cool-down phase. The women exercised at $\sim 50 \%-60 \%$ heart rate reserve (HRR), arrived at using the Karvonen formula, ${ }^{15}$ and gradually progressed in duration and intensity until they were able to exercise at $>85 \%$ HRR for 45 minutes. All the exercise sessions were supervised, and heart rates were monitored during exercise with chest-strap heart rate monitors (Polar Electro Inc., Lake Success, 
NY, USA). The average compliance with the exercise sessions and WL classes was $\sim 86 \%$.

\section{Procedures}

The maximal oxygen uptake $\left(\mathrm{VO}_{2} \max \right)$ was measured by indirect calorimetry, using a continuous graded treadmill test. ${ }^{16}$ The subjects' height $(\mathrm{cm})$ and weight $(\mathrm{kg})$ were measured to calculate BMI. The waist circumference was measured at the narrowest point, superior to the hip. The hip circumference was measured at the maximal circumference of the buttocks, typically in line with the pubic bone. The fat mass and lean tissue mass of the total body and the trunk were determined by DXA. The trunk area was defined, from below the chin to the top of the pelvis. A single CT scan, at the lumbar (L)4-L5 region, was used to determine the muscle area and the low-density lean tissue area (defined as intramuscular adipose tissue [IMAT]) of the trunk muscles. The erector spinae, psoas, lateral abdominal, and rectus abdominis muscles were isolated by manually drawing a line around each muscle. No bone density pixels were included in the muscle area. The Hounsfield unit (HU) was used to quantify the areas of muscle (30-80 HU) and IMAT (0-29 $\mathrm{HU})$ - the range of 0-29 HU for the measurement of the IMAT did not overlap the HU that corresponded to muscle. The trunk composition, as measured by CT, was analyzed using MIPAV (Medical Image Processing, Analysis and Visualization) v.7.0.0 software (NIH Center for Information Technology, Bethesda, MD, USA). First, the contrast of the image was manipulated using the toolbars, to ensure the best visibility of the muscles. Using the freehand tool, the right side of the first muscle group (erector spinae) was traced. Next, in the MIPAV tool bar, the plug-ins were selected (eg, $\rightarrow$ Algorithm $\rightarrow$ CT_MD). The output provided the results for the areas of fat, IMAT, and the high-density muscle, in $\mathrm{mm}^{2}$. The steps were repeated for the psoas and lateral abdominal muscles on the right side. Then, all steps were repeated on the left side for the psoas, lateral abdominal, and rectus abdominis. The areas of the right and left sides were averaged in these analyses. The entire rectus abdominis was traced and so no average was required. A test for reproducibility of the muscle area and IMAT measurements showed a coefficient of variation of less than $5 \%$ on repeat analysis.

\section{Statistical analyses}

A univariate analysis of variance (ANOVA) was used to test for the difference in the changes between the AEX+WL and WL groups. Within each intervention, the differences between the preintervention and postintervention measures of the variables were determined using a paired $t$-test. Pearson correlations analyses were used to determine the correlations between trunk body composition, age, and fitness. Statistical significance was set at $P<0.05$. The data were analyzed using SPSS statistical software (PASW Statistics for Windows, Version 18.0; SPSS Inc., Chicago, IL, USA) and expressed as mean \pm standard error of the mean (SEM).

\section{Results}

The demographic information was as follows: over $50 \%$ of the postmenopausal women were educated and had one or more college degrees (12\% bachelor, 33\% master, and 6\% doctorate degrees). Furthermore, 19\% had some college, while the remaining women had vocational training (3\%), an associate degree $(6 \%)$, high school degree $(6 \%)$, or education unknown (15\%). The majority of the women were currently married (63\%), and another $8 \%$ were widowed, $11 \%$ divorced, $13 \%$ never married, or, for $5 \%$, had an unknown marital status. In addition, $48 \%$ worked full-time and another $13 \%$ worked part time, $22 \%$ of women were retired, and $17 \%$ had an unknown employment status. About $37 \%$ of the women had annual incomes (in US\$) $>\$ 51,000$, and the remaining women had incomes of $\$ 41,000-50,000$ $(9 \%),<\$ 31,000-40,000(9 \%),<\$ 21,000-30,000(11 \%)$, $<\$ 11,000-20,000(8 \%),<\$ 11,000(3 \%)$, or had an unknown income $(23 \%)$.

\section{Response to $A E X+W L$ and $W L$}

The baseline characteristics were not different between the groups (Table 1). The $\mathrm{VO}_{2}$ max increased by $11 \%$ after the AEX+WL intervention $(1.77 \pm 0.07$ vs $1.97 \pm 0.08 \mathrm{~L} / \mathrm{min})$ $(P<0.001)$ but did not change with WL alone $(1.48 \pm 0.08$ vs $1.49 \pm 0.06 \mathrm{~L} / \mathrm{min})$ (group effect) $(P<0.001)$. There were no other significant differences between the interventions (no group effects) in the changes in body composition (Table 1). Body weight decreased $8 \%$ after both AEX+WL and WL $(P<0.001)$. Both the BMI and waist circumference decreased $(P<0.001)$, whereas the waist-to-hip ratio did not change after $\mathrm{AEX}+\mathrm{WL}$ or WL. The fat mass decreased by $15 \%$ and $12 \%$, after AEX+WL and WL, respectively $(P<0.001)$, with small but significant reductions in lean mass in both groups $(-2 \%$ in the AEX+WL group $[P<0.05]$ and $-4 \%$ in the WL group $[P<0.01])$. There was a decrease in trunk fat mass by $16 \%$ after AEX+WL $(P<0.001)$ and by $12 \%$ after WL $(P<0.001)$; the trunk lean mass was not significantly changed after either intervention. In all women combined $(n=65)$, there was a significant reduction in body weight, BMI, percent fat, fat mass, lean mass, and trunk fat mass (all $P<0.001$ ). 
Table I Body composition

\begin{tabular}{|c|c|c|c|c|c|c|}
\hline & \multicolumn{2}{|c|}{ AEX+WL $(n=43)$} & \multicolumn{2}{|c|}{ WL $(n=22)$} & \multicolumn{2}{|c|}{ Total group $(n=65)$} \\
\hline & Pre & Post & Pre & Post & Pre & Post \\
\hline Weight (kg) & $84 \pm 2$ & $77 \pm 2^{\ddagger}$ & $91 \pm 3$ & $84 \pm 3^{\ddagger}$ & $86 \pm 2$ & $79 \pm 2^{\ddagger}$ \\
\hline BMI $\left(\mathrm{kg} / \mathrm{m}^{2}\right)$ & $32 \pm 1$ & $29 \pm 1^{\ddagger}$ & $34 \pm 1$ & $3 I \pm I^{\ddagger}$ & $33 \pm 1$ & $30 \pm 1 \ddagger$ \\
\hline Waist (cm) & $95 \pm 2$ & $90 \pm 2^{\ddagger}$ & $98 \pm 2$ & $92 \pm 2^{\ddagger}$ & $96 \pm 2$ & $91 \pm 2^{\ddagger}$ \\
\hline Waist-hip ratio & $0.80 \pm 0.01$ & $0.80 \pm 0.01$ & $0.83 \pm 0.01$ & $0.82 \pm 0.02$ & $0.8 I \pm 0.01$ & $0.8 \mathrm{I} \pm 0.0 \mathrm{I}$ \\
\hline Percent body fat & $46.6 \pm 0.8$ & $42.6 \pm 1 . I^{\ddagger}$ & $48.7 \pm 0.9$ & $46.4 \pm I . I^{\ddagger}$ & $47.3 \pm 0.6$ & $43.9 \pm 0.8^{\ddagger}$ \\
\hline Fat mass $(\mathrm{kg})$ & $39.9 \pm 1.6$ & $33.8 \pm 1.6^{\ddagger}$ & $45.0 \pm 2.3$ & $39.6 \pm 2.2^{\ddagger}$ & $42.6 \pm 1.3$ & $35.8 \pm 1.4^{\ddagger}$ \\
\hline Lean mass (kg) & $42.3 \pm 1.0$ & $41.5 \pm 0.8 *$ & $43.7 \pm 1.3$ & $41.9 \pm 1.2^{\dagger}$ & $42.8 \pm 0.8$ & $41.6 \pm 0.7^{\ddagger}$ \\
\hline Fat-free mass (kg) & $45.0 \pm 1.0$ & $44.1 \pm 0.8^{*}$ & $46.5 \pm 1.3$ & $44.6 \pm 1.2^{\dagger}$ & $45.5 \pm 0.8$ & $44.3 \pm 0.7^{\ddagger}$ \\
\hline Trunk fat mass $(\mathrm{kg})$ & $19.1 \pm 0.8$ & $16.0 \pm 0.90 \ddagger$ & $21.9 \pm 1.1$ & $19.2 \pm 1.1^{\ddagger}$ & $20.1 \pm 0.7$ & $17.1 \pm 0.7^{\ddagger}$ \\
\hline Trunk lean mass (kg) & $20.6 \pm 0.5$ & $20.2 \pm 0.4$ & $20.0 \pm 1.2$ & $20.1 \pm 0.7$ & $20.4 \pm 0.5$ & $20.1 \pm 0.3$ \\
\hline
\end{tabular}

Notes: Values are mean \pm SEM. Significantly different pre- vs postintervention: $* P<0.05 ;{ }^{\dagger} P<0.0$ I; ${ }^{\ddagger} P<0.00$ I.

Abbreviations: AEX, aerobic exercise; BMI, body mass index; SEM, standard error of the mean; WL, weight loss; vs, versus.

Figure 1 is an example of the CT tracing analysis for the trunk muscle composition. The muscle area and IMAT area for the erector spinae, psoas, lateral abdominals, and rectus abdominis are presented in Table 2. In comparisons of the amount of IMAT relative to the amount of muscle in the women at baseline, the rectus abdominis IMAT area and rectus abdominis muscle area were relatively similar, indicating the highest fatty infiltration in this abdominal muscle. In contrast, the psoas muscle had the lowest relative amount ( $22 \%)$ of IMAT. The IMAT of the erector spinae and lateral abdominals was $\sim 35 \%-40 \%$ of the total area.

After AEX+WL, the IMAT decreased in the erector spinae $(-6 \%)(P<0.05)$, psoas $(-9 \%)(P<0.01)$, lateral abdominals $(-11 \%)(P<0.005)$, and rectus abdominis $(-9 \%)(P<0.05)$. After WL, the IMAT decreased in the lateral abdominal muscle $(-13 \%)(P<0.05)$. Although the IMAT decreased in the other muscles after WL, the changes were not statistically significant for the erector spinae $(-7 \%)$, psoas $(-11 \%)$, or rectus abdominis $(-3 \%)$. In terms of muscle area, both the erector spinae and the psoas muscle areas tended to decrease

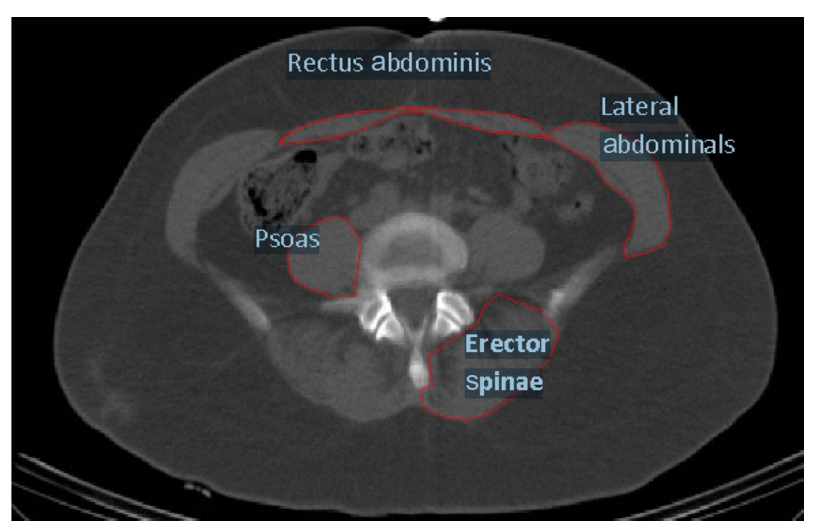

Figure I Ectopic fat in trunk muscles. after AEX+WL $(P=0.05)$. There was also a tendency for the erector spinae muscle area to decrease $(P=0.08)$ after $\mathrm{WL}$, but the psoas muscle area did not change. There was no significant change in either the lateral or rectus abdominal muscle areas after AEX+WL or WL.

There were no group effects for any changes in trunk composition. Thus, the absolute changes were not different after WL or AEX+WL in the IMAT or muscle areas for the erector spinae, psoas, lateral abdominal, or rectus abdominis muscles. When both groups were combined (total $\mathrm{n}=65$ ), the changes in each outcome were subsequently assessed. The IMAT significantly decreased in all four muscle groups (Figure 2), specifically, in the erector spinae by $6 \%(P=0.005)$, the psoas by $9 \%(P=0.007)$, the lateral abdominals by $11 \%(P=0.000)$, and in the rectus abdominis by $6 \%(P=0.045)$. The muscle area did not significantly change for the psoas or the lateral and rectus abdominal muscles, and decreased in the erector spinae $(-8 \%)(P=0.008)$.

With respect to the relationships with trunk composition, the erector spinae muscle area $(r=-0.43, P<0.001)$, psoas muscle area $(r=-0.29, P<0.05)$, and rectus abdominis muscle area $(r=-0.36, P<0.01)$ declined with age in the women. There was a tendency for the lateral abdominal muscle area to also decrease with age $(r=-0.24, P=0.05)$. At baseline, the spinal muscle areas were related to the $\mathrm{VO}_{2} \max$ (erector spinae muscle area $r=0.26, P<0.05$ and psoas muscle area $r=0.48, P<0.001)$. In addition, both the lateral abdominal muscle area $(r=0.40, P<0.005)$ and the rectus abdominal muscle area $(r=0.37, P<0.01)$ correlated with the $\mathrm{VO}_{2}$ max. The change in $\mathrm{VO}_{2}$ max with the interventions did not correlate with the change in trunk muscle area.

The IMAT for all four muscles was related to the total body fat mass (erector spinae $r=0.29, P<0.05$; psoas $r=0.45$, 
Table 2 Trunk composition

\begin{tabular}{|c|c|c|c|c|c|c|}
\hline & \multicolumn{2}{|c|}{ AEX+WL $(n=43)$} & \multicolumn{2}{|c|}{$W L(n=22)$} & \multicolumn{2}{|c|}{ Total group $(n=65)$} \\
\hline & Pre & Post & Pre & Post & Pre & Post \\
\hline \multicolumn{7}{|c|}{ Erector spinae area $\left(\mathrm{cm}^{2}\right)$} \\
\hline Muscle & $18.6 \pm 1.2$ & $17.3 \pm 1.3 * *$ & $16.6 \pm 1.3$ & $14.9 \pm 1.0 * * *$ & $17.9 \pm 0.9$ & $16.5 \pm 0.9^{\dagger}$ \\
\hline IMAT & $11.9 \pm 0.4$ & $11.2 \pm 0.5 *(n=42)$ & $11.9 \pm 1.0$ & $11.1 \pm 0.8$ & $11.9 \pm 0.4$ & $11.2 \pm 0.4^{\dagger}(n=64)$ \\
\hline \multicolumn{7}{|c|}{ Psoas area $\left(\mathrm{cm}^{2}\right)$} \\
\hline Muscle & $16.1 \pm 0.7$ & $15.4 \pm 0.7^{*}$ & $14.0 \pm 0.7$ & $14.3 \pm 0.8$ & $15.4 \pm 0.5$ & $15.0 \pm 0.6$ \\
\hline IMAT & $4.4 \pm 0.2$ & $4.0 \pm 0.2^{\dagger}$ & $4.5 \pm 0.4$ & $4.0 \pm 0.2$ & $4.6 \pm 0.2$ & $4.0 \pm 0.2^{\dagger}$ \\
\hline \multicolumn{7}{|c|}{ Lateral abdominal area $\left(\mathrm{cm}^{2}\right)$} \\
\hline Muscle & $19.1 \pm 0.9$ & $18.7 \pm 1.0(n=42)$ & $15.4 \pm 0.9$ & $15.7 \pm 1.0$ & $17.8 \pm 0.7$ & $17.6 \pm 0.7(n=64)$ \\
\hline IMAT & $11.4 \pm 0.6$ & $10.3 \pm 0.6^{\dagger}(n=42)$ & $12.7 \pm 1.0$ & $11.0 \pm 0.8 *$ & $11.8 \pm 0.5$ & $10.6 \pm 0.5^{\ddagger}(n=64)$ \\
\hline \multicolumn{7}{|c|}{ Rectus abdominis area $\left(\mathrm{cm}^{2}\right)$} \\
\hline Muscle & $4.1 \pm 0.4$ & $3.7 \pm 0.3(n=42)$ & $2.9 \pm 0.4$ & $3.3 \pm 0.4$ & $3.6 \pm 0.3$ & $3.6 \pm 0.3(n=64)$ \\
\hline IMAT & $3.2 \pm 0.2$ & $2.9 \pm 0.2 *(n=42)$ & $3.6 \pm 0.4$ & $3.5 \pm 0.4$ & $3.4 \pm 0.2$ & $3.1 \pm 0.2 *(n=64)$ \\
\hline
\end{tabular}

Notes: Values are mean \pm SEM. Significantly different pre- vs postintervention: $* P<0.05 ;{ }^{\dagger} P<0.01 ;{ }^{\ddagger} P<0.001 ; * * P=0.05 ; * * * P=0.08$.

Abbreviations: AEX, aerobic exercise; IMAT, intramuscular adipose tissue; SEM, standard error of the mean; WL, weight loss; vs, versus.

$P<0.001$; lateral abdominals $r=0.59, P<0.001$; and rectus abdominis $r=0.48, P<0.001)$. In addition, the muscle area of the psoas, and lateral and rectus abdominals correlated with the total body lean mass $(r=0.30, r=0.27$, and $r=0.35$, $P<0.05)$. The changes in body weight related to changes of the erector spinae, lateral abdominal, and rectus abdominis IMAT (Table 3). The change in body weight did not correlate with changes in muscle area. The loss of fat mass was related to the loss of IMAT of the erector spinae and the lateral abdominals (Table 3 ). The changes in trunk fat mass by DXA did not correlate with the changes in IMAT for any muscle.

\section{Discussion}

The present investigation was designed to test the association between fitness and trunk muscle areas and to determine whether fatty infiltration of the trunk muscles can be reduced with lifestyle changes in overweight and obese

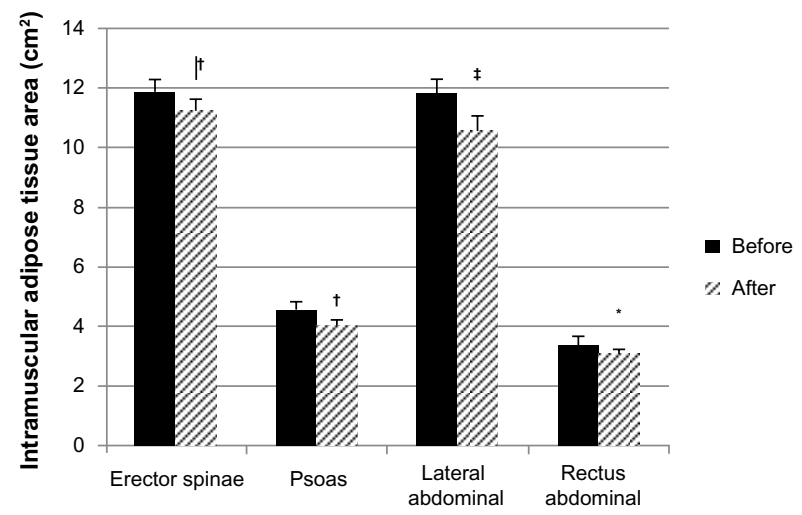

Figure 2 Intramuscular adipose tissue area of the spine and abdominal muscles before and after the interventions.

Notes: $* P<0.05 ;{ }^{\dagger} P<0.01 ; \ddagger P<0.001$. older women. The major novel findings were that a lifestyle modification of diet-induced WL, alone or with AEX training, resulted in a significant reduction of the fat infiltration in the abdominal and spinal muscles of the trunk region. The relationship between the muscle area in these muscles and $\mathrm{VO}_{2}$ max suggests a role of physical fitness in the development or maintenance of the abdominal and spinal muscles. Our finding that losses of total body fat predict a reduction in IMAT of the trunk support the idea that WL reduces fat depots throughout the body.

Aging is associated with a loss of muscle and increased fat infiltration in the muscles of the thigh in women. ${ }^{17}$ The increase in fat infiltration in the trunk muscles with age observed in this cohort of postmenopausal women parallels the increase in IMAT we have previously reported in the thigh muscles in women aged 18-70 years. Our data over a 30-year span suggests that within the postmenopausal period, there is also an increase in trunk ectopic fat. It is interesting to note that the rectus abdominal muscle had the greatest fatty infiltration, followed by the lateral abdominal muscle and erector spinae, in these overweight and obese women, suggesting that obesity contributes to the increased IMAT in these muscles. In fact, there was almost an equal amount of fat and muscle in the rectus abdominis. The psoas

Table 3 Predictors of changes in IMAT

\begin{tabular}{lll}
\hline & $\Delta$ body weight & $\Delta$ fat mass \\
\hline Erector spinae IMAT area & $0.25^{*}$ & $0.27^{*}$ \\
Psoas IMAT area & 0.11 & 0.09 \\
Lateral abdominal IMAT area & $0.24^{*}$ & $0.31^{*}$ \\
Rectus abdominis IMAT area & $0.27^{*}$ & 0.15 \\
\hline
\end{tabular}

Note: Significance $* P<0.05$.

Abbreviation: IMAT, intramuscular adipose tissue. 
muscles had the lowest IMAT relative to the amount of muscle. A possible explanation for the low IMAT in the psoas is that it is an active muscle that is recruited bilaterally to stabilize the lumbar spine ${ }^{18}$ and flex the hip. ${ }^{19}$ These findings would suggest the need to emphasize the strengthening of the abdominal muscles through exercise, to increase the muscle area in this region, in postmenopausal women.

Older women have an increased risk and greater propensity for obesity-related loss of function. The trunk extensors are required to stabilize the trunk in forward motion. ${ }^{20}$ In postmenopausal women with osteoporosis, back extensor strength and lumbar spinal mobility are significant predictors of quality of life. ${ }^{21}$ Trunk extensor endurance has been associated with dynamic performance, as measured by the Short Physical Performance Battery and Berg Balance in community-dwelling older adults. ${ }^{22}$ In the same group, trunk extensor strength was associated with static balance. ${ }^{22}$ These studies ${ }^{21,22}$ provide evidence to illustrate the importance of the trunk musculature in mobility and balance. Even though only women who were sedentary, with low levels of fitness, were included in our study, we were able to demonstrate that each of the muscle areas was related to the $\mathrm{VO}_{2} \max$ at baseline.

The clinical significance of poor trunk composition is illustrated by the associations between reduced muscle attenuation and physical function. Increased muscle fat infiltration has been shown to be related to lower knee extensor muscle strength as well as to future mobility limitations among well-functioning older individuals. ${ }^{23,24}$ IMAT is also related to mobility levels as tested by 6-minute walk time, stair ascent and descent time, and timed get-up-and-go in community ambulating older men and women who had two or more comorbid disease conditions and who were at risk for falling. ${ }^{25}$ Moreover, the association of increased trunk muscle fat infiltration with functional limitations in older adults is independent of thigh muscle fat infiltration. ${ }^{7}$ Hicks et al measured muscle attenuation in the lumbar paraspinal, lateral abdominal, and rectus abdominis muscles of over 1,500 men and women in the Health Aging and Body Composition (ABC) study to show that trunk muscle attenuation was a strong predictor of physical function scores ${ }^{8}$ as well as standing and dynamic balance. Moreover, trunk muscle attenuation was found to be a significant predictor of function in the group with the greatest severity of back pain. The authors suggested that rehabilitation efforts in individuals with higher back pain may need to address the paraspinal and lateral abdominal muscles. Furthermore, a reduced spine density or low muscle attenuation, reflective of increased fat within the erector spinae, was related to kyphosis in older men and women. ${ }^{9}$ Although we did not have a measure of back pain or classification of kyphosis, our finding of a reduction of the fat infiltration in these same muscle groups by weight reduction could suggest that WL may also help as an effective rehabilitation strategy in those with back pain.

We believe that these are the first data to provide evidence that WL can reduce ectopic fat in trunk muscles. The decrease in IMAT, by CT, was relatively similar across the erector spinae, psoas, rectus abdominis and lateral abdominal muscles, and between groups. The slightly greater decrease in the lateral abdominal IMAT is likely to have been due to the relatively larger amount of IMAT compared with muscle area. We also determined loss of fat mass in the trunk region by DXA. Our average $12 \%-16 \%$ loss of fat in the trunk region, by DXA, over the 6-month period is similar to the loss observed in the visceral or subcutaneous abdominal fat area $(-13 \%$ and $-12 \%){ }^{12}$ Moreover, it is similar to changes in muscle attenuation of the midthigh, ${ }^{12}$ which is known to be important in lower extremity strength. Our correlation of the changes in body weight and changes in low-density lean tissue of the erector spinae, psoas, and lateral and rectus abdominal muscles suggests that greater WL is associated with the loss of intramuscular fat. Since the treadmill intervention in this study did not specifically train any of these trunk muscles, we did not expect to see muscle hypertrophy in these muscles. Future studies could address abdominal muscle weakness in postmenopausal women and integrate core exercises to improve abdominal and spinal muscle composition in these individuals.

There are several limitations of this study that are worth mentioning. The results of this study are applicable to older women. However, it is likely that WL interventions in older men would result in a similar reduction in fat infiltration in the muscles of the spine and abdomen. Although we found reductions in the erector spinae and psoas IMAT after WL that were similar in magnitude to those in the AEX+WL group, the smaller sample size in the WL group may have limited the detection of statistical significance for this group. Although there were no group effects for any changes in trunk composition, we cannot determine without an AEXonly (no WL) group whether exercise is necessary for reducing ectopic fat in the trunk muscles. The use of a single CT slice may not capture all the adaptations that occur in the trunk and may also overestimate the amount of fat relative to muscle in this one area compared with the whole muscle group. Another limitation was the lack of a measure of balance and strength, to examine whether the changes in 
trunk composition translated to an improvement in these measures. It would also have been valuable to understand whether the reductions in fatty infiltration are maintained if weight is regained. The strengths of our study include the novelty of the aims, the longitudinal clinical design, carefully controlled interventions and use of in vivo CT methodology for the assessment of body composition, and the inclusion of an at-risk study population of overweight and obese older women.

In conclusion, weight reduction programs, alone or with aerobic exercise, reduced fat infiltration in four muscles of the trunk, the erector spinae, psoas, and lateral and rectus abdominal muscles, and decreased the erector spinae muscle area. Furthermore, the reduction in body weight and loss of total body fat was associated with the reduction of ectopic fat in the trunk muscles. These observations, together with the age-associated loss of muscle area in the trunk, in postmenopausal women, provide additional support for the view that WL and exercise targeted at these muscles are important recommendations to consider for the physical function and mobility in older women.

\section{Acknowledgments}

Dr Ryan is supported by a Veterans Affairs Research Career Scientist Award and the National Institute of Health (NIH) (grant RO1-AG-030075); this research was supported by the Baltimore Veterans Affairs Medical Center Geriatric Research, Education and Clinical Center (GRECC), by the NIH (grants R01-AG-019310, RO1-AG-20116) and the General Clinical Research Center (GCRC) (\#M01 RR016500), the Nutrition Obesity Research Center (NORC) of Maryland (DK072488), and by the University of Maryland Claude D Pepper Older Americans Independence Center (grant P30-AG-12583).

Our appreciation is extended to the women who participated in this study. We are grateful to Dr Goldberg, the nurses, research assistants, and dieticians of the Division of Gerontology and Geriatric Medicine and GRECC, for their assistance with this project.

\section{Disclosure}

The authors report no conflicts of interest in this work.

\section{References}

1. Hedley AA, Ogden CL, Johnson CL, Carroll MD, Curtin LR, Flegal KM. Prevalence of overweight and obesity among US children, adolescents, and adults, 1999-2002. JAMA. 2004;291(23):2847-2850.

2. Fried LP, Bandeen-Roche K, Chaves PH, Johnson BA. Preclinical mobility disability predicts incident mobility disability in older women. J Gerontol A Biol Sci Med Sci. 2000;55(1):M43-M52.
3. Launer LJ, Harris T, Rumpel C, Madans J. Body mass index, weight change, and risk of mobility disability in middle-aged and older women. The epidemiologic follow-up study of NHANES I. JAMA. 1994;271(14): 1093-1098.

4. Jensen GL, Friedmann JM. Obesity is associated with functional decline in community-dwelling rural older persons. J Am Geriatr Soc. 2002;50(5):918-923.

5. Houston DK, Ding J, Nicklas BJ, et al; Health ABC Study. Overweight and obesity over the adult life course and incident mobility limitation in older adults: the health, aging and body composition study. Am J Epidemiol. 2009;169(8):927-936.

6. Ryan AS, Nicklas BJ, Berman DM. Racial differences in insulin resistance and mid-thigh fat deposition in postmenopausal women. Obes Res. 2002;10(5):336-344.

7. Hicks GE, Simonsick EM, Harris TB, et al. Cross-sectional associations between trunk muscle composition, back pain, and physical function in the health, aging and body composition study. J Gerontol A Biol Sci Med Sci. 2005;60(7):882-887.

8. Hicks GE, Simonsick EM, Harris TB, et al. Trunk muscle composition as a predictor of reduced functional capacity in the health, aging and body composition study: the moderating role of back pain. J Gerontol A Biol Sci Med Sci. 2005;60(11):1420-1424.

9. Katzman W, Cawthon P, Hicks GE, et al. Association of spinal muscle composition and prevalence of hyperkyphosis in healthy communitydwelling older men and women. J Gerontol A Biol Sci Med Sci. 2012;67(2):191-195.

10. Sinaki M, Brey RH, Hughes CA, Larson DR, Kaufman KR. Balance disorder and increased risk of falls in osteoporosis and kyphosis: significance of kyphotic posture and muscle strength. Osteoporos Int. 2005;16(8):1004-1010.

11. Ryan SD, Fried LP. The impact of kyphosis on daily functioning. $J$ Am Geriatr Soc. 1997;45(12):1479-1486.

12. Ryan AS, Ortmeyer HK, Sorkin JD. Exercise with calorie restriction improves insulin sensitivity and glycogen synthase activity in obese postmenopausal women with impaired glucose tolerance. Am J Physiol Endocrinol Metab. 2012;302(1):E145-E152.

13. Lichtenstein AH, Appel LJ, Brands M, et al. Summary of American Heart Association Diet and Lifestyle Recommendations revision 2006. Arterioscler Thromb Vasc Biol. 2006;26(10): 2186-2191.

14. Harris JA, Benedict FG. A Biometric Study of Basal Metabolism in Man. Washington, DC: Carnegie Institute of Washington; 1919.

15. Karvonen MJ, Kentala E, Mustala O. The effects of training on heart rate; a longitudinal study. Ann Med Exp Biol Fenn. 1957;35(3): 307-315.

16. Ryan AS, Nicklas BJ, Berman DM. Aerobic exercise is necessary to improve glucose utilization with moderate weight loss in women. Obesity (Silver Spring). 2006;14(6):1064-1072.

17. Ryan AS, Nicklas BJ, Elahi D. A cross-sectional study on body composition and energy expenditure in women athletes during aging. Am J Physiol. 1996;271(5 Pt 1):E916-E921.

18. Hu H, Meijer OG, van Dieën JH, et al. Is the psoas a hip flexor in the active straight leg raise? Eur Spine J. 2011;20(5):759-765.

19. Park RJ, Tsao H, Claus A, Cresswell AG, Hodges PW. Changes in regional activity of the psoas major and quadratus lumborum with voluntary trunk and hip tasks and different spinal curvatures in sitting. J Orthop Sports Phys Ther. 2013;43(2):74-82.

20. Leteneur S, Gillet C, Sadeghi H, Allard P, Barbier F. Effect of trunk inclination on lower limb joint and lumbar moments in able men during the stance phase of gait. Clin Biomech (Bristol, Avon). 2009;24(2): 190-195.

21. Miyakoshi N, Hongo M, Maekawa S, Ishikawa Y, Shimada Y, Itoi E. Back extensor strength and lumbar spinal mobility are predictors of quality of life in patients with postmenopausal osteoporosis. Osteoporos Int. 2007;18(10):1397-1403.

22. Suri P, Kiely DK, Leveille SG, Frontera WR, Bean JF. Trunk muscle attributes are associated with balance and mobility in older adults: a pilot study. $P M$ R. 2009;1(10):916-924. 
23. Schaap LA, Koster A, Visser M. Adiposity, muscle mass, and muscle strength in relation to functional decline in older persons. Epidemiol Rev. Epub December 4, 2012.

24. Visser M, Goodpaster BH, Kritchevsky SB, et al. Muscle mass, muscle strength, and muscle fat infiltration as predictors of incident mobility limitations in well-functioning older persons. J Gerontol A Biol Sci Med Sci. 2005;60(3):324-333.
25. Marcus RL, Addison O, Dibble LE, Foreman KB, Morrell G, Lastayo P. Intramuscular adipose tissue, sarcopenia, and mobility function in older individuals. J Aging Res. 2012;2012:629637.

\section{Publish your work in this journal}

Clinical Interventions in Aging is an international, peer-reviewed journal focusing on evidence-based reports on the value or lack thereof of treatments intended to prevent or delay the onset of maladaptive correlates of aging in human beings. This journal is indexed on PubMed Central, MedLine, the American Chemical Society's 'Chemical Abstracts
Service' (CAS), Scopus and the Elsevier Bibliographic databases. The manuscript management system is completely online and includes a very quick and fair peer-review system, which is all easy to use. Visit http://www.dovepress.com/testimonials.php to read real quotes from published authors.

Submit your manuscript here: http://www.dovepress.com/clinical-interventions-in-aging-journal 